\title{
A two-parameter analysis of surface fatigue crack growth behavior of fine-grained WC-Co cemented carbide at different stress ratios
}

\author{
Hiroko Mikado ${ }^{1, *}$, Sotomi Ishihara $^{2,3}$, Noriyasu Oguma $^{3}$ and Shingo Kawamura ${ }^{1}$ \\ ${ }^{1}$ Department of Machinery and Engineering Group, YKK Corporation, Kurobe, Toyama, 938-8601, Japan \\ ${ }^{2}$ National Institute of Technology, Toyama College, Hongo, Toyama, 939-8630, Japan \\ ${ }^{3}$ Department of Mechanical Engineering, University of Toyama, Gofuku, Toyama, 930-8555, Japan
}

\begin{abstract}
In this study, the fatigue crack growth (FCG) characteristics of the short surface crack was studied at various stress ratios, $R$, using the fine grained cemented carbide. The FCG law for the material was studied using two parameters, the maximum stress intensity factor $K_{\max }$ and the stress intensity factor range $\Delta K$. It was found that the relationship between the rate of FCG, $\mathrm{d} a / \mathrm{d} N$ and $\Delta K$ depends on the $R$ value. In the $\mathrm{d} a / \mathrm{d} N$ vs. $K_{\max }$ relation, at the region of high crack growth rate, the FCG rate was nearly constant by the value of $K_{\max }$ without depending on $R$. However, in the low FCG rate region, the FCG rate tended to accelerate as $R$ became lower. Therefore, it was difficult to specify $\mathrm{d} a / \mathrm{d} N$ as a single value at different $R$ ratios using either $\Delta K$ or $K_{\max }$ from the low speed region to the high-speed region. For that reason, the FCG behaviour of the material was studied based on the crack growth law, $\mathrm{d} a / \mathrm{d} N=\mathrm{A} K_{\max }{ }^{m} \Delta \mathrm{K}^{n}$. The values of $m$ and $n$ were obtained as 6.6 and 1.4, respectively. Therefore, it was found that the FCG rate of the cemented carbide was strongly dependent on $K_{\max }$ rather than $\Delta K$, like the FCG characteristics of ceramics.
\end{abstract}

\section{Introduction}

Cemented carbide is hard material and has excellent wear resistance, so it has been widely used in cutting tools and so on. In recent years, cemented carbide has been increasingly used for the mold material. In the forging process, compressive stress and shear stress are repeatedly applied to the mold material at the boundary between the work and the mold.

In the above situation, short surface fatigue cracks initiate and grow on the mold, causing chipping and fracture of the mold. It is expected that the initiation and growth behavior of the short surface crack are concerned with wear behavior of the mold [1]. Currently, how to extend the mold life has been a big issue for manufacturing companies.

Researches on the fatigue crack growth (FCG) behavior of the cemented carbide have been mainly conducted targeting the long through crack.

Moreover, these studies were mainly conducted in the high $K_{\max }$ area where the FCG speed is faster than $10^{-9} \mathrm{~m} /$ cycle or more. For example, Fry et al. [2] studied the FCG behavior of the long through crack, using various cemented carbides. They reported the effect of the WC grain size and Co content on the FCG characteristics of these cemented carbides. In addition, Llanes et al. [3, 4] reported the FCG characteristics of the long through crack of the various cemented carbides.
Both of the above studies focused on the high-speed FCG zone, larger than $10^{-9} \mathrm{~m} /$ cycle.

On the other hand, there have been few researches on the FCG behavior for the short surface crack, due to difficulty in doing experiments and observations. Ishihara et al. [5] reported the FCG characteristics of the short surface crack at various stress ratios using the cemented carbide with comparatively large WC grain size. Mikado et al. [6] studied the FCG behavior of the short surface crack at stress ratio, $R=-1$ using the finegrained cemented carbide.

In this study, the FCG behavior of the short surface crack was investigated at various stress ratios using the fine grain cemented carbide. In particular, the FCG behavior was studied in the low FCG zone below $10^{-9}$ $\mathrm{m} /$ cycle, which is important for practical use and has not been clarified so far.

The FCG data obtained were evaluated using two parameters, the maximum stress intensity factor $K_{\max }$ and the stress intensity factor range $\Delta K$.

In the two parameters analysis of FCG, the FCG behavior of the short surface were compared with those of the long penetrating crack. Then, the characteristics of FCG behavior of the short surface crack were clarified. Consideration was also given how the prediction accuracy in the crack growth amount was changed depending on whether FCG threshold is taken into consideration or not. 


\section{Two-parameter analysis}

FCG behavior of the short surface crack for the fine grain cemented carbide were analysed using two parameters of $K_{\max }$ and $\Delta K$ [7]. In the FCG law using two parameters, the following equation [7] will be used. Here, $m$ and $n$ are constants determined from experiments. The decision method for the values of $m$ and $n$ will be described later.

$$
\mathrm{d} a / \mathrm{d} N=\mathrm{CK}_{\max }{ }^{m} \Delta K^{n}
$$

The following equation holds between $K_{\max }$ and $\Delta K$, where $R$ is the stress ratio, $K_{\min }$ and $K_{\max }$ are the minimum stress intensity factor and the maximum stress intensity factor, respectively.

$$
\begin{aligned}
& K_{\max }=\Delta K /(1-R) \\
& R=K_{\min } / K_{\max }
\end{aligned}
$$

Substituting equation (2) into equation (1) and transform it, we obtain the following relation.

$$
\mathrm{d} a / \mathrm{d} N /(1-R)^{n}=\mathrm{C} K_{\max }^{m+n}
$$

The constants $m$ and $n$ in equation (3) were determined as follows.

First, FCG data obtained from experiments at various stress ratios $R$ was plotted on the $\log -\log$ graph. In that case, the vertical axis was $\mathrm{d} a / \mathrm{d} N /(1-R)^{n}$ and the horizontal axis was taken as $K_{\max }$. The first $n$ value used was arbitrary assumed. After that, the $n$ value was varied with a step width of 0.1. Multiple log $\left(\mathrm{d} a / \mathrm{d} N /(1-R)^{n}\right)-\log K_{\max }$ relationships were created for different $n$ values.

Thereafter, on the logarithmic graph, the above relation was approximated by a straight line having a slope $(m+$ $n$ ). The approximation degree of these lines to the experimental data was judged from the value of correlation coefficient.

The relation of $\log \left(\mathrm{d} a / \mathrm{d} N /(1-R)^{n}\right)-\log K_{\max }$ where the correlation coefficient becomes maximum was determined, and $m$ and $n$ values at that time were obtained.

\section{Material and experimental procedures}

\subsection{Material}

In the present study, a commercially available finegrained WC-Co cemented carbide was used as the testing material. Tables 1 and 2 show the chemical composition and mechanical properties of the material, respectively. The material contains $13.0 \mathrm{wt} \% \mathrm{Co}$ and its bending strength is $4100 \mathrm{MPa}$. Figure 1 shows the microstructure of the material, which is a composite microstructure consisting of both $\mathrm{WC}$ and Co phase. A detailed microstructural analysis of Fig. 1 revealed that the material has a log-normal distributed WC grains with a modal value of about $0.35 \mu \mathrm{m}$, and has an exponential distributed binder mean free paths, ranging from 0 to 1 $\mu \mathrm{m}$.

Table 1. Chemical composition of the WC-Co cemented carbide $[\mathrm{wt} \%]$.

\begin{tabular}{|c|c|c|}
\hline Co & Cr & W and C \\
\hline 13.0 & 0.51 & Bal. \\
\hline
\end{tabular}

Table 2. Mechanical properties of the WC-Co cemented carbide.

\begin{tabular}{|c|c|c|c|}
\hline $\begin{array}{c}\text { Young's } \\
\text { modulus } \\
{[\text { GPa ] }}\end{array}$ & $\begin{array}{c}\text { Fracture } \\
\text { toughness, } \boldsymbol{K}_{\text {IC }} \\
\text { [ MPam }^{\mathbf{2} \text { [ }} \text { ] }\end{array}$ & $\begin{array}{c}\text { Bending } \\
\text { strength } \\
{[\text { MPa ] }}\end{array}$ & $\begin{array}{c}\text { Vickers } \\
\text { hardness } \\
{[\text { HV ] }}\end{array}$ \\
\hline 550 & 12.1 & 4100 & 1480 \\
\hline
\end{tabular}

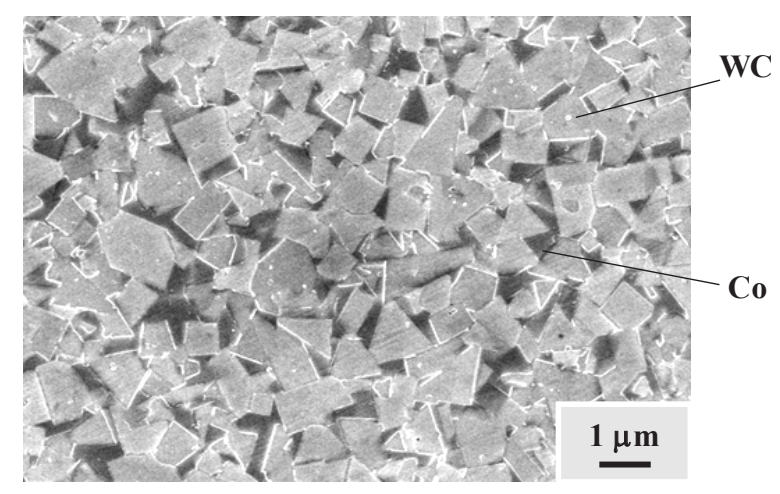

Fig. 1. Microstructure of the WC-Co cemented carbide used in the study.

\subsection{Specimens}

The specimen used in the present study was a rectangular shaped specimen having a width of $8 \mathrm{~mm}$, a height of $4 \mathrm{~mm}$, and a length of $50 \mathrm{~mm}$, as shown in Fig. 2. A Vickers indentation (Indentation load: $400 \mathrm{~N}$ and indentation time $20 \mathrm{sec}$ ) was introduced on the centre of specimens to make a pre-crack.

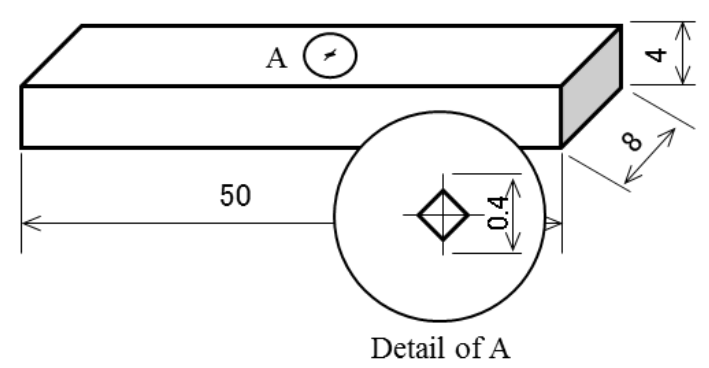

Fig. 2. Specimen used for investigating the FCG rate of short surface cracks at $R=0.1,0.2$ and 0.5 . 


\subsection{Experimental Procedures}

Four-point bending fatigue tests (inner span: $10 \mathrm{~mm}$, outer span: $30 \mathrm{~mm}$ ) were conducted at three different stress ratios, $R=0.1,0.2$ and 0.5 , using the rectangular specimens. To eliminate the effects of the indentation, such as those of machining and residual stress, on the FCG behaviour of short surface cracks, the pre-crack was extended to a length of approximately $200 \mu \mathrm{m}$ by applying a cyclic load. After that, crack growth measurement was started.

A servo-hydraulic fatigue testing machine was used to perform the four-point bending fatigue test. Tests were conducted in laboratory air at room temperature with a stress frequency of $10 \mathrm{~Hz}$. Crack length during the fatigue process was measured using the replication technique [8].

For calculation of the maximum stress intensity factor $K_{\max }$, equation (4) was used assuming that the crack shape was a semicircular.

$$
K_{\max }=Y \sigma_{\max } \sqrt{\pi a}
$$

Here, $Y$ is a correction coefficient $(=0.73), \sigma_{\max }$ is the maximum stress, and $a$ is a half crack length. Equation (5) was used for calculation of the stress intensity factor range $\Delta K$.

$$
\begin{aligned}
& \Delta K=K_{\max }-K_{\min }\left(K_{\min }>0\right), \\
& \Delta K=K_{\max }\left(K_{\min } \leq 0\right)
\end{aligned}
$$

\section{Experimental results}

\subsection{FCG curve at various stress ratios}

In this study, FCG behavior of the fine grain cemented carbide was investigated under various stress ratios. To examine the FCG curve, two types of experiments, $\sigma_{\max }$ constant test and $K_{\max }$ constant test, were performed. Some of the experimental results are shown in Fig. 3. Fig. 3(a) and Figs. 3(b) - 3(d) show the test results at the $\sigma_{\max }$-constant condition and the $K_{\max }$-constant condition, respectively. In the former, the approximate curve to the experimental data was drawn, and the rate of FCG, $\mathrm{d} a / \mathrm{d} N$, was determined from the approximate curve. In the constant $K_{\max }$ test, the $2 a-N$ curve obtained under the constant $K_{\max }$ condition was approximated by the straight line and the rate of FCG, $\mathrm{d} a / \mathrm{d} N$, was measured from its slope. The maximum stress intensity factor, $K_{\max }$ and the stress intensity factor range, $\Delta K$ were evaluated using the equations (4) and (5), respectively.

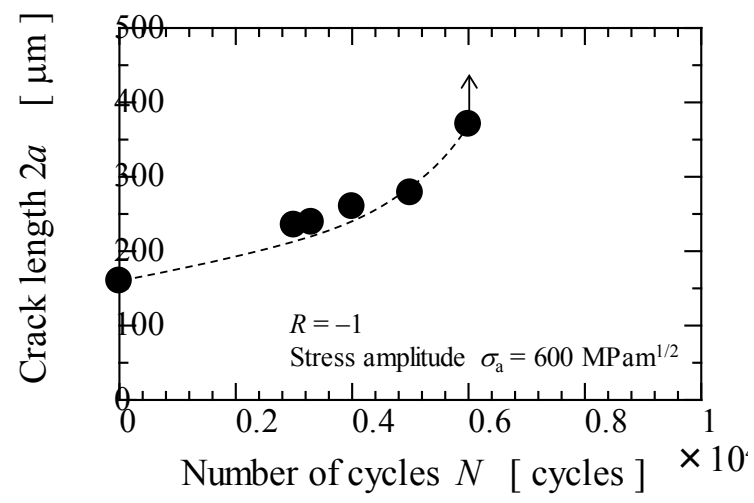

(a) Stress amplitude $=$ constant, $R=-1$ [5]

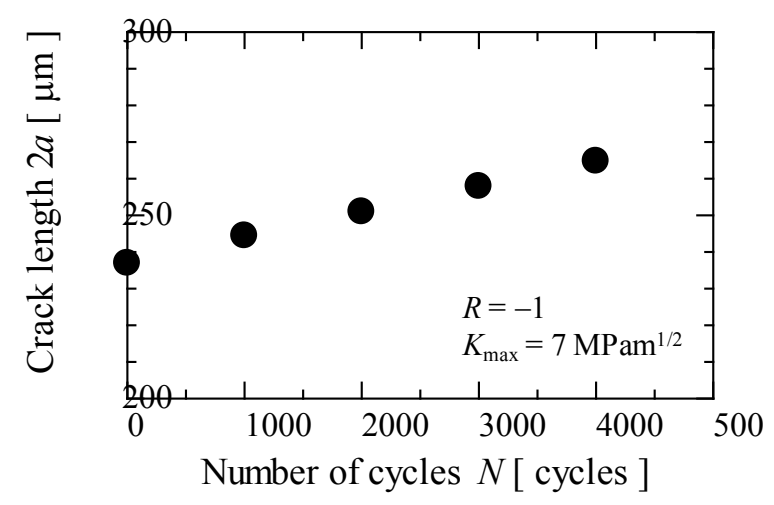

(b) $K_{\max }=$ constant, $R=-1$

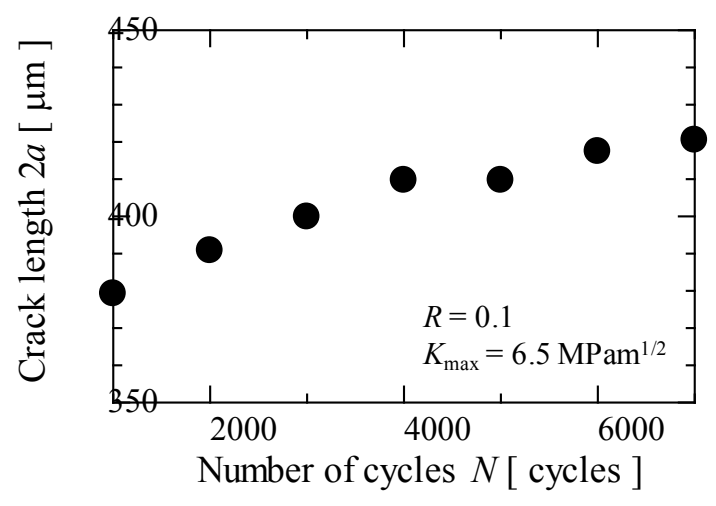

(c) $K_{\max }=$ constant, $R=0.1$

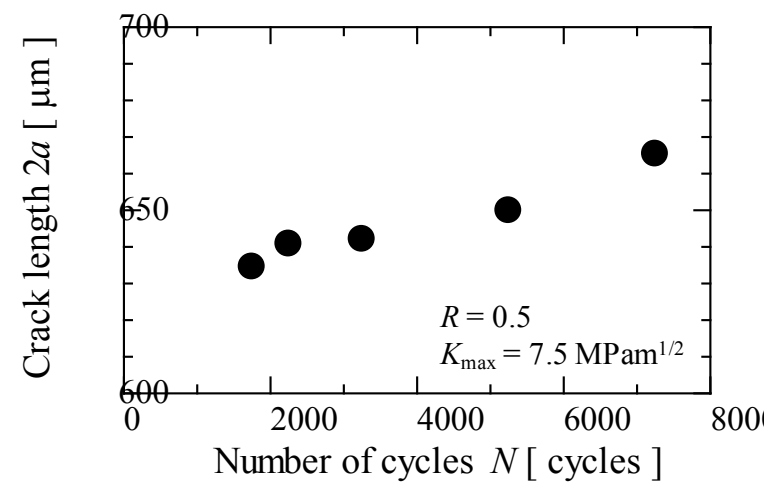

(d) $K_{\max }=$ constant, $R=0.5$

Fig. 3. Crack growth curve at different stress ratios. 


\subsection{FCG law using one parameter and two parameter}

\subsubsection{One parameter analysis}

From the FCG curve described in section 4.1, the rate of FCG, $\mathrm{d} a / \mathrm{d} N$ was obtained. The relations, $\mathrm{d} a / \mathrm{d} N$ vs. $K_{\max }$ and $\mathrm{d} a / \mathrm{d} N$ vs. $\Delta K$, are shown in Figs. 4 (a) and 4 (b), respectively.

As can be seen from Fig. 4 (a), at the high $K_{\max }$ region where $\mathrm{d} a / \mathrm{d} N$ is larger than $5 \times 10^{-9} \mathrm{~m} /$ cycle, $\mathrm{d} a / \mathrm{d} N$ can be determined by the $K_{\max }$ value regardless of the $R$ ratio. On the other hand, looking at the $\mathrm{d} a / \mathrm{d} N-\Delta K$ relationship in Fig. 4 (b), $\mathrm{d} a / \mathrm{d} N$ is not uniquely determined by $\Delta K$ and varies depending on the $R$ ratio. More specifically, the necessary $\Delta K$ for a constant FCG rate, $\mathrm{d} a / \mathrm{d} N$, decreases as the $R$ ratio increases. This tendency is particularly conspicuous in the low FCG region less than $10^{-9} \mathrm{~m} /$ cycle.

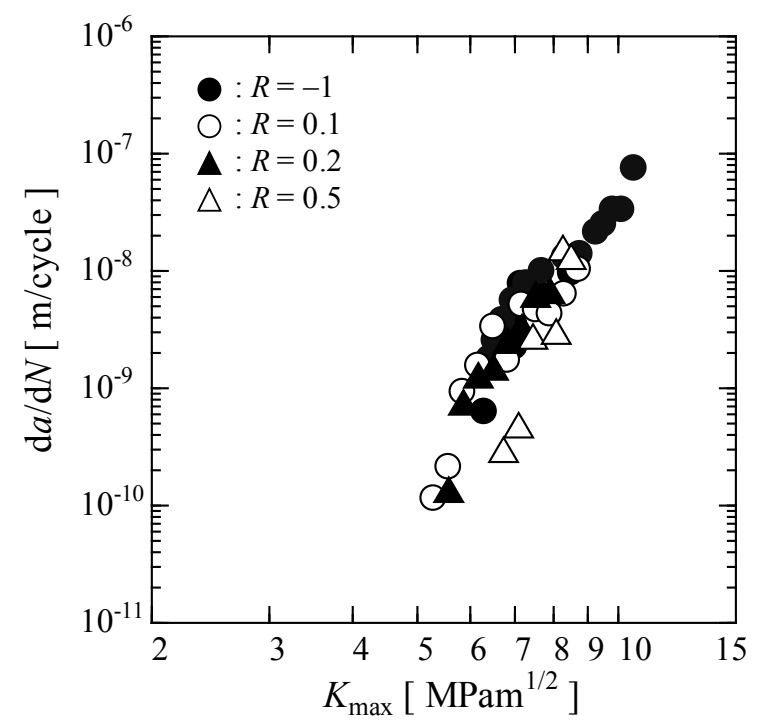

(a) Relationship between $\mathrm{d} a / \mathrm{d} N$ and maximum stress intensity $K_{\max }$ for the four different stress ratios.

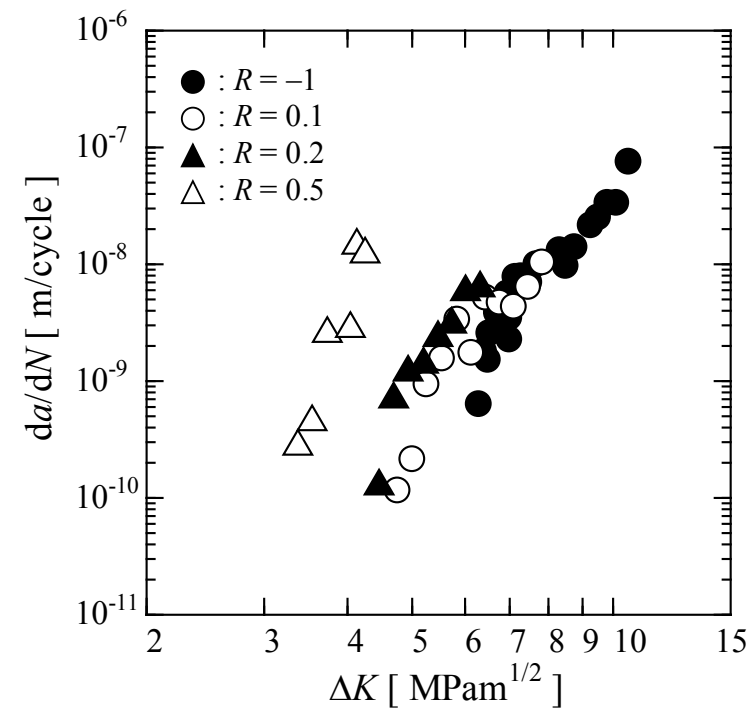

(b) Relationship between $\mathrm{d} a / \mathrm{d} N$ and stress intensity factor range $\Delta K$ for the four different stress ratios.

Fig. 4. Crack growth curve at different stress ratios.
The relationships $\mathrm{d} a / \mathrm{d} N-K_{\max }$, and $\mathrm{d} a / \mathrm{d} N-\Delta K$ of the above fine grained cemented carbide show the FCG characteristics similar to ceramics [7] rather than metal. Therefore, the FCG law using both parameters of $K_{\max }$ and $\Delta K$ will be examined next.

\subsubsection{Two parameter analysis}

FCG law expressed by equation (3) using both parameters of $K_{\max }$ and $\Delta K$ will be applied to the present experimental data. Figure 5 shows the log-log plot of the FCG data for the fine grain cemented carbide at various stress ratios $R$. The vertical and horizontal axises of the figure represent $\mathrm{d} a / \mathrm{d} N /(1-R)^{n}$ and $K_{\max }$, respectively.

For the experimental data of $R=-1$, it was assumed that stress fluctuation on the compressive stress side do not affect the FCG behavior and was handled as experimental result of $R=0$.

Figure 5 shows the relationship between $\mathrm{d} a / \mathrm{d} N /(1-R)^{n}$ and $K_{\max }$ for the fine cemented carbide. In Fig. 5, the values of $m=6.6$ and $n=1.4$ were used. These values were determined using the maximum correlation coefficient method described in Chapter 2. As can be seen from the figure, experimental data of various stress ratios can be approximated by the straight line in the figure.

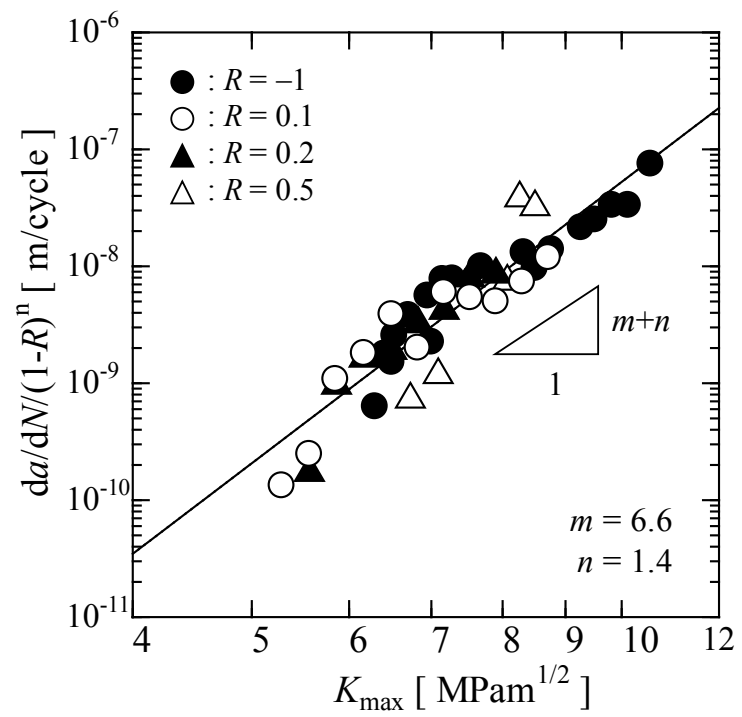

Fig. 5. Relationship between $\mathrm{d} a / \mathrm{d} N /(1-R)^{n}$ and $K_{\max }$ of the fine cemented carbide.

\section{Discussion}

\subsection{Comparison with the FCG behaviour of long through cracks}

Llanes et al. [3] investigated the FCG behavior of through cracks using the cemented carbide (WC grain size: $0.5 \mu \mathrm{m}, \mathrm{Co}: 16.3 \%, K_{\mathrm{IC}}: 9.2 \mathrm{MPam}^{1 / 2}$ ) of the same grade as the one used in this study. In the following, the FCG characteristics of the short-surface cracks observed in this study and the one for the long through cracks 
obtained by Llanes et al. [3] were compared with each other.

However, the experimental result of Llanes et al. is targeting to a high-speed region where the FCG rate is $10^{-9}$ to $10^{-6} \mathrm{~m} /$ cycle. On the other hand, in the shortsurface crack of this study, the low velocity region of $10^{-10}$ to $10^{-7} \mathrm{~m} /$ cycle, including the threshold level of the FCG, is targeted. So, there is a difference in the measurement area.

Figure 6 shows a log-log plot of the relationship between $\mathrm{d} a / \mathrm{d} N /(1-R)^{n}$ and $K_{\max }$ for the long through crack obtained by Llanes et al. Values of $m$ and $n$ for the long through crack obtained were 22 and 5, respectively. These values are obviously high compared with $m$ value 6.6 and $n$ value 1.4 for the short-surface crack. On the other hand, the ratio $n / m$ of both shows the same value about 0.21 .

Therefore, as compared with the short-surface cracks, the long through cracks are highly susceptible to $K_{\max }$ and $\Delta K[6]$, and shows FCG characteristics of brittle materials such as ceramics.

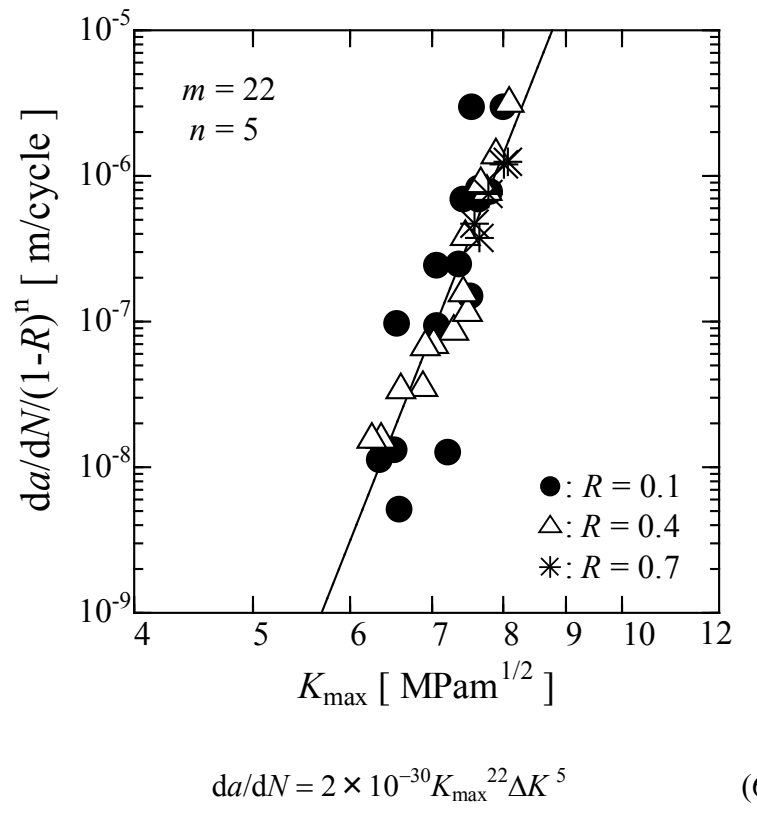

Fig. 6. Relationship between $\mathrm{d} a / \mathrm{d} N /(1-R)^{n}$ and $K_{\max }$ obtained by Llanes et al. [3]. The data was obtained for the long through crack using the fine grained cemented carbide.

\subsection{FCG law considering the threshold level of crack growth}

To accurately evaluate the rate of FCG in the low speed region, it is considered necessary to introduce the threshold level of crack growth, $K_{\mathrm{th}}$. Therefore, in this study, the threshold levels of crack growth, $K_{\text {max,th }}$ were determined from the experimental data, by defining $K_{\text {max,th }}$ as the values for $K_{\max }$ at the speed, $\mathrm{d} a / \mathrm{d} N=10^{-10}$ $\mathrm{m} /$ cycle. The value of $\Delta K_{\text {th }}$ can be obtained from the value of $K_{\max , \text { th }}$ using the relation, $\Delta K_{\mathrm{th}}=(1-R) K_{\mathrm{max}, \text { th }}$.
Table 3. Threshold level of crack growth at each stress ratio.

\begin{tabular}{|c|c|c|c|c|}
\hline $\begin{array}{c}\text { Stress } \\
\text { ratio, } \boldsymbol{R}\end{array}$ & $\mathbf{- 1}(\mathbf{0})$ & $\mathbf{0 . 1}$ & $\mathbf{0 . 2}$ & $\mathbf{0 . 5}$ \\
\hline$K_{\text {max,th }}$ & 5.3 & 5.3 & 5.6 & 6.6 \\
\hline$\Delta K_{\text {th }}$ & 5.3 & 4.8 & 4.5 & 3.3 \\
\hline
\end{tabular}

Table 3 shows the values of $K_{\max \text {,th }}$ and $\Delta K_{\text {th }}$ obtained for each stress ratio $R$. As can be seen from the table, as $R$ increases, $K_{\max , \text { th }}$ increases and conversely $\Delta K_{\text {th }}$ decreases. With cemented carbide, it is considered that WC grains form crack bridging portions [9]. This crack bridging locally reduces the driving force of fatigue crack [10]. As the $R$ value decreases, the stress variation range increases. In the case, it is considered that elimination of the bridging portion will be promoted. Conversely, as the $R$ value increases, the elimination of the bridging portion does not proceed, and as a result, it is considered that $K_{\max \text {,th }}$ increases.

By substituting these values into the following equation, $K_{\text {max }}^{*}$ and $\Delta K^{*}$ can be obtained. The FCG law using $K^{*}{ }_{\max }$ and $\Delta K^{*}$ instead of $K_{\max }$ and $\Delta K$ was derived.

$$
\begin{gathered}
\Delta K_{\max }^{*}=K_{\max }-K_{\text {max,th }} \\
\Delta K^{*}=\Delta K-\Delta K_{\text {th }}=\Delta K-(1-R) K_{\text {max,th }}
\end{gathered}
$$

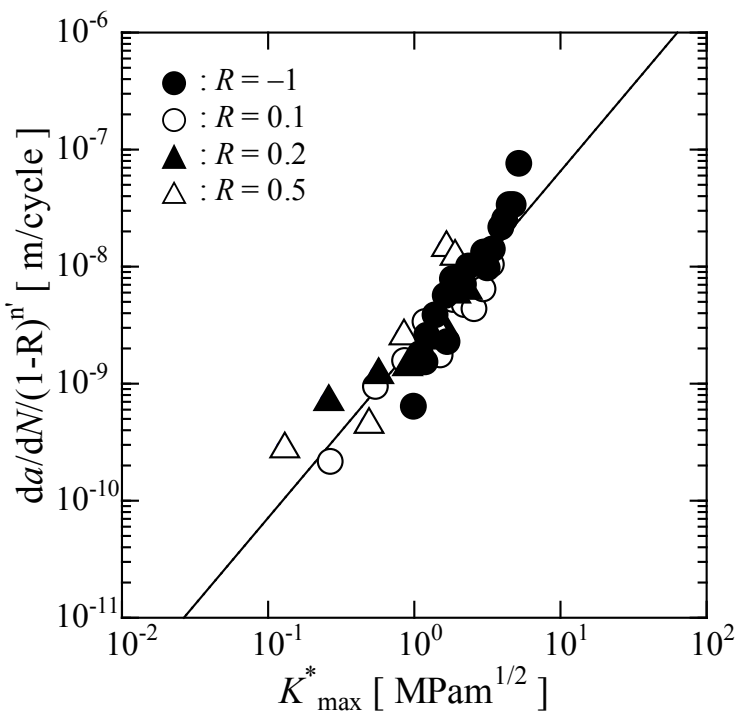

Fig. 7. Relationship between $\mathrm{d} a / \mathrm{d} N /(1-R)^{n^{\prime}}$ and $K_{\text {max }}^{*}$ of the cemented carbide used in the present study.

Figure 7 shows the log-log plot for the relationship between $\mathrm{d} a / \mathrm{d} N /(1-R)^{n^{\prime}}$ and $K_{\max }^{*}$ of the short surface crack. Compared with the $\mathrm{d} a / \mathrm{d} N /(1-R)^{n}-K_{\max }$ relation in Fig. 5, in the low FCG speed range of Fig. 7 , it is found that the fitness with experimental data and FCG law is clearly improved. The $\mathrm{m}^{\prime}$ and $\mathrm{n}^{\prime}$ values obtained 
were 1.5 and 0 , respectively. These values are lower than the $\mathrm{m}$ and $\mathrm{n}$ values in Table 3 .

In addition, by introducing the threshold levels of crack growth into the FCG law, $\mathrm{d} a / \mathrm{d} N-K_{\max }^{*}{ }^{m^{\prime}} \Delta K^{*^{\prime} n^{\prime}}$, the value of $n^{\prime}=0$ was obtained. So, the FCG law with one parameter $K_{\text {max }}^{*}$ was obtained. This result is different from the research result of Llanes et al. As one of the reasons for this, their research may be directed to the high-speed regions that do not need to consider FCG threshold levels.

\subsection{Prediction accuracy of FCG amount}

Next, the FCG speed under various stress ratios was estimated using the obtained FCG law. A comparison between the estimation result and the experimental one is shown in Fig. 8. As can be seen from the figure, the estimation result from the FCG law using two parameters corresponds well with the experimental result.

Moreover, it can be seen that the estimation accuracy of the crack growth amount is higher when the $K_{\text {max,th }}$ is introduced into FCG law than the case when FCG law does not take $K_{\max , \text { th }}$ into consideration. Also, when dealing with FCG behavior of cemented carbide at various stress ratios, it is necessary to know whether only $K_{\max }$ parameter is sufficient, or, two parameters of $K_{\max }$ and $\Delta K$ are necessary. To make above issue clear in future, a systematic study including other cemented carbides is necessary. In addition, it is necessary to consider the physical meaning of the $m^{\prime}, n^{\prime}$ values.

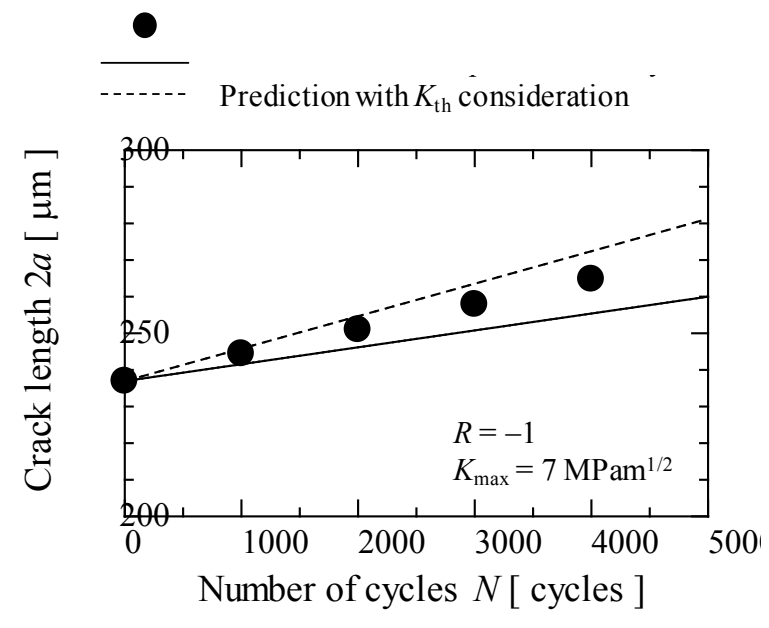

(a) $K_{\max }=$ constant, $R=-1$

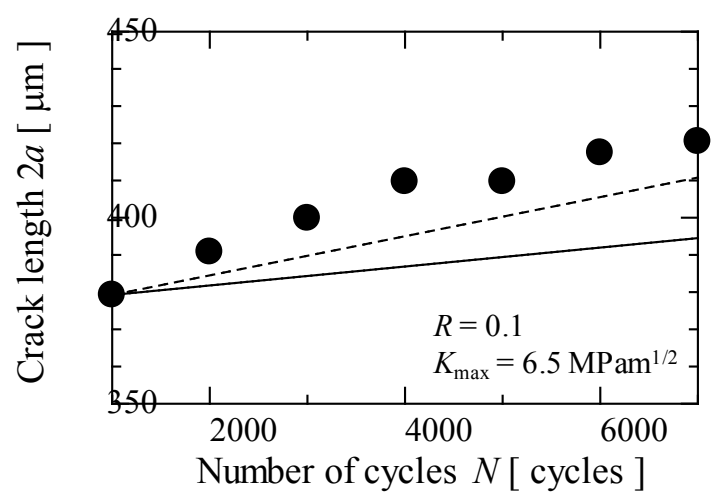

(b) $K_{\max }=$ constant, $R=0.1$

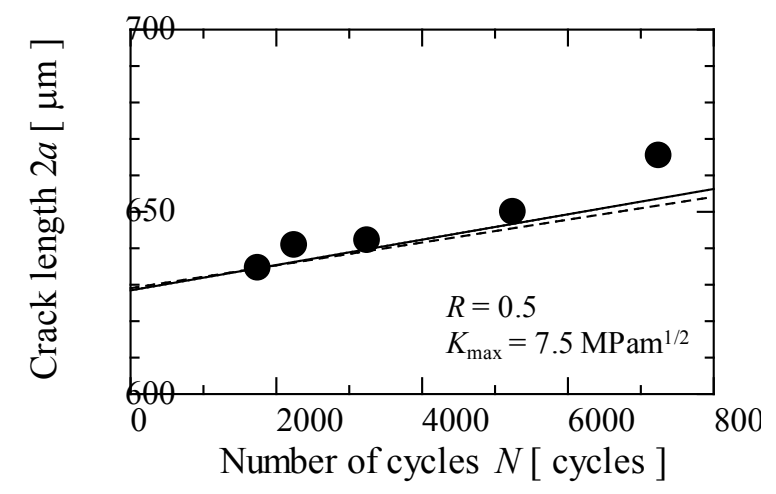

(c) $K_{\max }=$ constant, $R=0.5$

Fig. 8. Comparison between estimated results and experimental results for the crack growth rate.

\section{Concluion}

The short surface FCG behavior for the fine grained cemented carbide was studied under different stress ratios. The obtained experimental data was analysed using two parameters, $K_{\max }$ and $\Delta K$, and the following results were obtained.

(1) In the relationship $\mathrm{d} a / \mathrm{d} N-K_{\max }$, in the high $K_{\max }$ region, $\mathrm{d} a / \mathrm{d} N$ was determined by the $K_{\max }$ value regardless of the $R$ ratio, but in the low $K_{\max }$ region, the influence of the $R$ ratio appeared. On the other hand, in the $\mathrm{d} a / \mathrm{d} N-\Delta K$ relationship, $\mathrm{d} a / \mathrm{d} N$ was not uniquely determined by $\Delta K$ but depends on the $R$ ratio.

(2) The short-surface FCG law using two parameters $K_{\max }$ and $\Delta K$ of the cemented carbide was expressed by $\mathrm{d} a / \mathrm{d} N=\mathrm{A} K_{\max }{ }^{m} K^{n}$. Here $m$ and $n$ are material constants, and their values were 6.6 and 1.4 , respectively. Therefore, FCG rate of cemented carbide strongly depends on $K_{\max }$ rather than $\Delta K$, indicating the brittle FCG characteristics like ceramics.

(3) In comparison with the FCG law of the long through crack obtained by Llanes et al., the $m$ and $n$ values for the short surface crack were found to be lower than those of the long through crack. However, the $n / m$ ratio was comparable. Therefore, the FCG law obtained for the long through crack cannot be applied to that of the short surface crack. 
(4) By introducing the threshold levels, $K_{\max \text {,th }}$ and $\Delta K_{\text {th }}$ into the relation $\mathrm{d} a / \mathrm{d} N-K_{\max }^{m} \Delta K^{n}$, the value of $n^{\prime}=$ 0 was obtained. So, the FCG law with one parameter $K_{\max }$ was obtained.

(5) In the FCG law of the short surface crack using two parameters, when the $K_{\text {max,th }}$ was introduced into FCG law, the estimation accuracy of the crack growth amount was higher than the case when the FCG law did not take $K_{\text {max,th }}$ into consideration.

\section{Acknowledgments}

Authors would like to express their sincere appreciation to the students of University of Toyama who helped us when carrying out the experiment.

\section{References}

1. A. Mizoguchi, T. Goshima, M. Shimizu, S. Ishihara, J. Sol. Mech. Mater. Eng. 2, 1080 (2008)

2. P.R. Fry, G.G. Garret, J. Mat. Sci. 23, 7, 2325 (1988)

3. L. Llanes, Y. Torres, M. Anglada, Acta Mater. 50, 2381 (2002)

4. Y. Torres, J. M. Tarrago, D. Coureaux, E. Tarrés, B. Roebuck, P. Chan, M. James, B. Liang, M. Tillman, R.K. Viswanadham, K.P. Mingard, A. Mestra, L. Llanes, Int. J. Refract. Met. Hard Mater. 45, 179 (2014)

5. S. Ishihara, H. Shibata, T. Goshima, M. Shimizu, J. Thermal Stresses, 31, 1025 (2008)

6. H. Mikado, S. Ishihara, N. Oguma, S. Kawamura, Metals, 7, 7 (2017)

7. R.O. Ritchie, Int. J. Fract. 100, 55 (1999)

8. S. Ishihara, A.J. McEvily, Proceeding of 12th International Conference on Fracture, CD-ROM (Ottawa, 2009)

9. H. Mikado, S. Ishihara, N. Oguma, K. Masuda, S. Kawamura, Fatigue of Materials III Advances and Emergences in Understanding: Proceedings of the Third Biennial Symposium, (Pittsburgh, 2014)

10. A.J. McEvily, R.O. Ritchie, Fatig. Fract. Eng. Mater. Struct. 21, 847 (1998) 